\title{
Construction of the fast neutron monitoring detectors for the COSINE-100 experiment
}

\author{
Govinda Adhikari* ${ }^{*}$ \\ Department of Physics, Sejong University, Seoul 05006, Republic of Korea E-mail: \\ adhikari.astrodgmail.com
}

\begin{abstract}
Measurements of the environmental neutron flux in the vicinity of dark matter search experiments are important because signals induced by these neutrons can mimic those that are expected from dark matter interactions. In order to establish a systematic understanding of the environmental neutron flux at the location of the COSINE-100 experiment, we developed a liquid scintillator neutron detector and studied its pulse shape discrimination capabilities and background contamination levels. In this paper, the construction of the neutron monitoring detector is presented.
\end{abstract}

The 39th International Conference on High Energy Physics (ICHEP2018)

4-11 July, 2018

Seoul, Korea

* Speaker.

${ }^{\dagger}$ COSINE-100 Collaborator 


\section{R\&D for fast neutron monitoring detector (NMD)}

A cylindrical vessel having 5-cm length and $4.5-\mathrm{cm}$ inner diameter is made of $1.5-\mathrm{cm}$ thick teflon is used to test the pulse shape discrimination (PSD) power and internal background of the target material. Two different LS, Linear Alkyl Benzene (LAB) and "Ultima Gold-F (UG-F)" based on Di-isopropylnaphthalene (DIN; $\mathrm{C}_{16} \mathrm{H}_{20}$ ) are used for this study. The neutron- $\gamma$ separation at the energy range of $[0.2,1.0]$ MeVee for the LAB based LS is measured $\sim 3 \sigma$ while UGF is $\sim 7 \sigma$. So, UG-F, is the candidate material for the NMD.

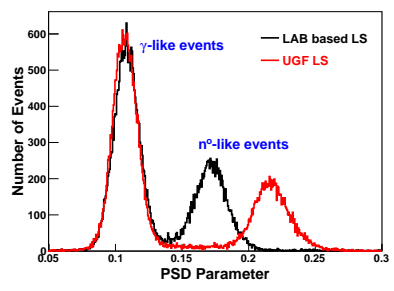

(a)

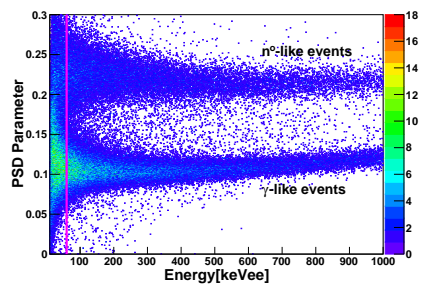

(b)

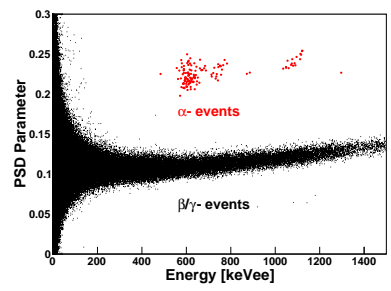

(c)

Figure 1: (a) Comparison of PSD parameter and scatter plot between PSD parameter and electron equivalent energy in case of the (b) ${ }^{252} \mathrm{Cf}$ neutron source and (c) background data.

This detector is installed inside the KIMS shielding facility [1]. The total $\alpha$ background is $(0.21 \pm 0.03) \mathrm{mBq} / \mathrm{kg}$ and dominant component is the ${ }^{210} \mathrm{Po}$ as shown in Fig.1.

\section{Construction of the NMD}

The NMD is constructed by using $\sim 5.5 \mathrm{~kg}$ of the UGF. Two 5 " hamamastsu photomultiplier tubes are coupled at both ends. We achieve PSD threshold between gamma and neutron events $\sim 0.3 \mathrm{MeVee}$ with dynamic range upto $\sim 10 \mathrm{MeVee}$ and having similar alpha background as obtained into the R\&D set-up [2]. This detector is installed at COSINE-100 experiment room [3] and neutron flux measurement is ongoing.

\section{Summary}

The DIN-based LS fast neutron detector is constructed and installed at the COSINE-100 experimental room. The PSD power $\sim 4 \sigma$ at the energy range of [0.3, 10.0] MeVee and internal alpha background is measured to be $(0.17 \pm 0.005) \mathrm{mBq} / \mathrm{kg}$. The neutron flux measurement is ongoing at the COSINE-100 experimental room.

\section{References}

[1] H. S. Lee et al., Development of low background CsI(Tl) crystals for WIMP search, Nuclear Instruments and Methods in Physics Research A, 571 (2007) 644-650.

[2] G. Adhikari et al., Study of fast neutron detector for COSINE-100 experiment, JINST 13 (2018) T06005.

[3] G. Adhikari et al., Initial performance of the COSINE-100 experiment, Eur. Phys. J. C 78 (2018) 107. 